\title{
An In-situ Method for Comparing Thermal Conductivity of Insulation Film in MEMS
}

\author{
Zhe Liu, a , Jiangbo Luo ${ }^{2, b}$ and Guifu Ding ${ }^{3, c}$ \\ 1,2,3 National Key Laboratory of Science and Technology on Micro/Nano Fabrication, \\ School of Electronic Information and Electrical Engineering, \\ Shanghai Jiao Tong University, Shanghai, 200240, P. R. China \\ a1143409010@sjtu.edu.cn, b luojiangbo@sjtu.edu.cn, c gfding@sjtu.edu.cn
}

Keywords: Infrared Imager, Thermal conductivity, AIN /PI, MEMS

Abstract. A effective method for comparing the thermal conductivity of insulation film was developed here. The thermal simulation on Ansys software indicated that different materials at same thickness exhibit different temperature distribution in this system. Base on this, the new measurement structure was fabricated by MEMS process. The results by Infrared Imager exhibited similar trends with the simulation. So the AIN/Polyimide nanocomposites film at different AlN content are compared based on this. The result shows that the AlN/PI at content(40\%) has the high thermal conductivity. What's more, this method can effectively and conveniently In-situ compare the thermal conductivity of insulation film in MEMS.

\section{Introduction}

The Micro-electromechanical Systems (MEMS) industry has been rapidly developed in recent years. The performance and reliability of these MEMS devices are influenced often by the degree to which they can conduct heat[1,2]. And thermal conductivity of thin films materials is an important parameter for many novel MEMS applications such as through silica via (TSV) in 3D packaging.

The thermal conductivity of thin film materials can change significantly from that of the corresponding bulk material. The difference in thermal conductivity between bulk materials and thin film materials is for the fact that for the film with small thicknesses[3-4]. So how to meausure the film conductivity is great problem. And there are little reports about film conductivity In-Situ measurement.

In this paper, a new method for comparing the thermal conductivity of insulation film in MEMS was developed. The simulation for measurement was performed on Ansys software. Base on simulation, the measure structure were fabricated by micro-machining process. And the AlN/Polyimide nanocomposites [5-7] at different AIN content are compared at this In-situ measurement method.

\section{Theory and Simulation}

The measurement principle is shown as figure 1. And the Pt resistor was used to be the heat source to heat the AIN/PI film. The PI below the Pt resistor was used as thermal insulation layer. So the most heat generated by Pt micro-heater were transferred to AlN/PI film layer and then converted with ambient air. In this measurement system, the heat generated by Pt heater keep same at different process, which can be achieved by controlling its' supply power, while the AlN/PI layer are varied with different AlN content. But all of different AIN/PI films are same in film thickness. And this can easily be achieved by spinning coating where the spinning speed or times are varied. 


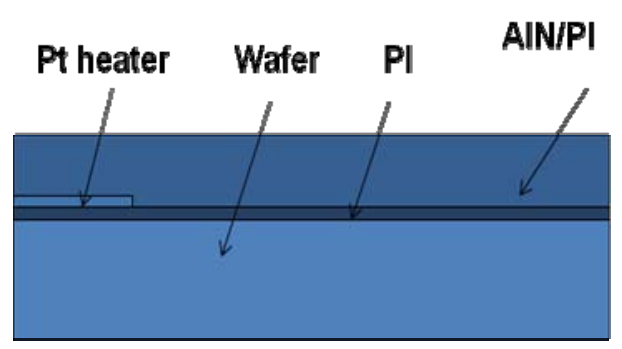

Figure1.the measurement principle

The thermal simulation was performed on the finite element software (Ansys 14.0). The element Solid 185 was used to analyze this system. The other parameters in model are very similar with actual parameters.

The simulation results are shown as figure 2(a). Obviously, the AlN/PI above the Pt heater are high in temperature and the film far away from heater are low in temperature. It conformed to theory of heat transfer. However, different content of AlN/PI film exhibits different trends in the simulation. The thermal conductivity of material were set as $(0.3 \mathrm{~W} / \mathrm{m} \cdot \mathrm{K}, 0.6 \mathrm{~W} / \mathrm{m} \cdot \mathrm{K}, 0.9 \mathrm{~W} / \mathrm{m} \cdot \mathrm{K}, 1.2 \mathrm{~W} / \mathrm{m}$. $\mathrm{K}, 1.5 \mathrm{~W} / \mathrm{m} \cdot \mathrm{K}, 3 \mathrm{~W} / \mathrm{m} \cdot \mathrm{K})$. From figure $2(\mathrm{~b})$, the high thermal conductivity material behave higher temperature than those which has low conductiviy above the Pt heater. And when the distance between the film and Pt heater become larger, the temperature decreased gradually. It's easy to find that the high thermal conductivity behave lower temperature at edge, while the low thermal conductivity behave higher temperature.

So from the simulation, the subsequent process to compare the conductivity of different materials which are in same thickness were plausible. The comprehensive process will be explained in next section.
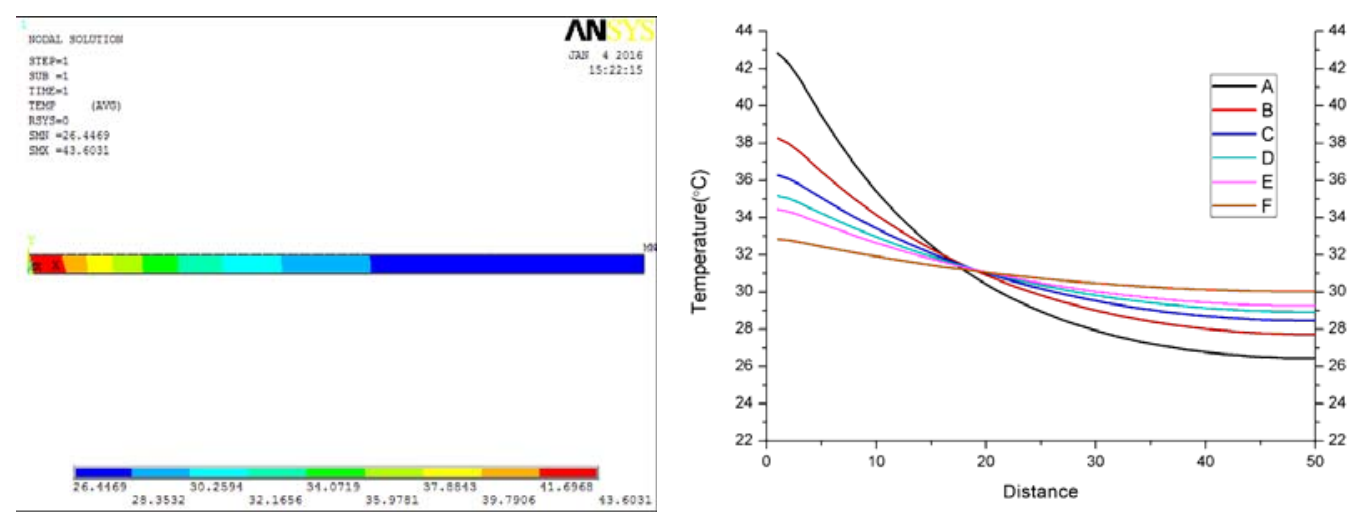

Figure 2. the simulation results (a)the temperature distribution at A,(b)relationship of temperature against distance at different thermal conductivity. From A to F, conductivity is from $0.3 \mathrm{~W} / \mathrm{m}$. K to 3 $\mathrm{W} / \mathrm{m} \cdot \mathrm{K}$.

\section{Experimental}

Base on the above simulation, such fabrication preocesses were made to effectively compare the film conductivity.And the fabrication process is shown as follows:

(a) The PI resin was spinning coating on wafer (3in) and the film are $20 \mathrm{um}$ in thickness.

(b)An 500nm pt was sputtered on the wafer.

(c)A $10 \mu \mathrm{m}$ positive photoresist (PR) of AZ 4620 was spin coated on a glass wafer as a sacrificial layer, the photoresist was dried at $110{ }^{\circ} \mathrm{C}$ for $1 \mathrm{~h}$.

(d)Then it was patterned by UV-lithography and developed. The patterned photoresist was obtained. 
(e) The Pt film on the wafer was etched, and the photoresist was cleaned by $\mathrm{NaOH}$ solvent (2\%) ,as is shown at figure $3(\mathrm{a})$.

(f)The AlN/PI precursor with a $50 \mu \mathrm{m}$ thickness was spin coated on wafer, then the wire was fabricated as figure $3(b)$.

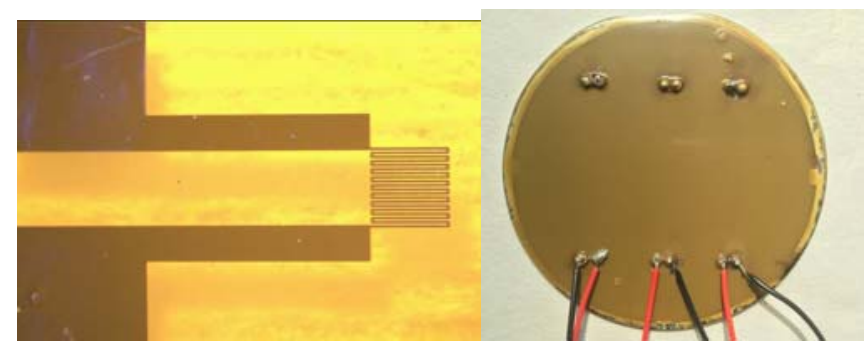

Figure 3. (a) the bare pt at microscope (b) the whole wiring of the measurement

The measurement is shown as figure 4 . The supply voltage was set at $2.3 \mathrm{~V}$, while the current was set at $0.37 \mathrm{~A}$. Infrared Imager was used to record the temperature distribution of wafer surface. For comparing different thermal conductivity, the power must keep same at the different measurement.

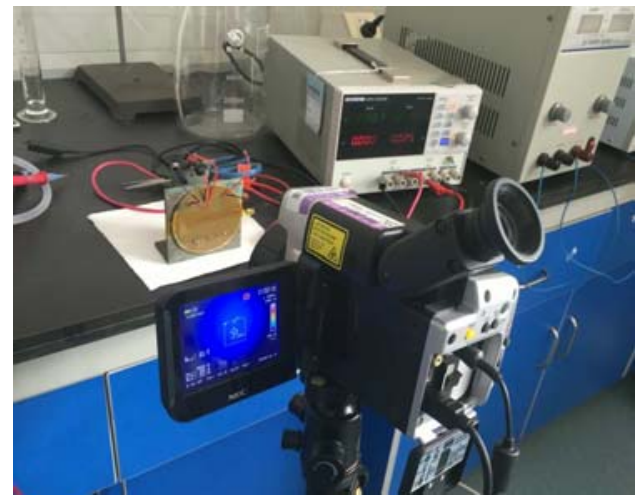

Figure 4 the measure process of Infrared Imager.

The measuring results are shown as figure 5. As is shown, the AlN/PI at $40 \%$ AlN content showed higher temperature in the center. According to simulation, this indicates that the AlN/PI nanocomposite film at $40 \%$ content has high thermal conductivity.

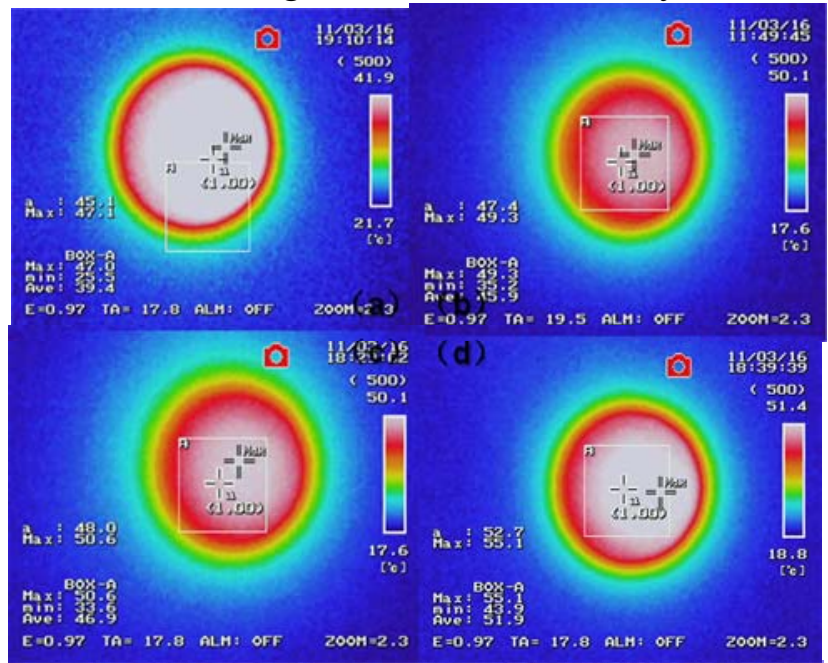

Figure 5 The measuring result(a)pure PI (b)8\%AlN/PI(c)20\%AlN/PI(d)40\%AlN/PI

The data obtained by Infrared Imager were analyzed with Infrared Imager Analyzer NS9500. The result is show as figure 6 , the curve are similar with the simulation. And the $40 \%$ AlN/PI film 
behave higher temperature than others above the pt heater. And the surface temperature decreased obviously, when the distance between the film and Pt heater become larger. It's easy to find that the $40 \%$ AlN/PI behave lower temperature at edge, while others behave higher temperature. All in all, with the AIN content increasing in PI matrix, the conductivity has been improved obviously.

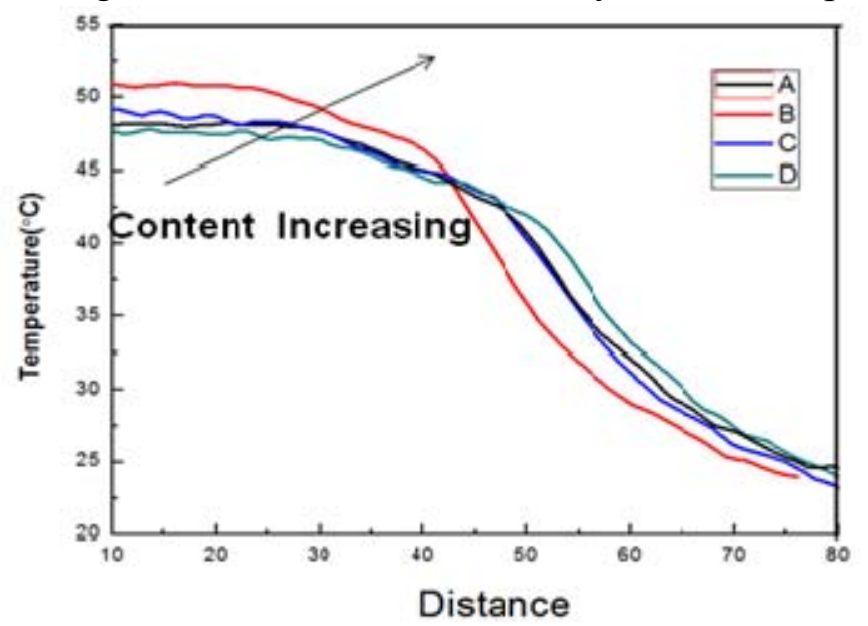

Figure 6 the relationships of temperature again distance at different AlN content

\section{Conclusions}

We developed an effective In-situ method for comparing the thermal conductivity of insulation film in MEMS. The thermal simulation performed on Ansys software indicated that different material at same thickness exhibit different surface temperature distribution. And this distribution was related to film conductivity. Base on this, the measurement structure was fabricated by MEMS process. The result by Infrared imager showed similar trend with the simulation. So we use this to compare the AlN/Polyimide nanocomposite films at different AlN content. The result showed that the AIN/PI at content $(40 \%)$ has the high thermal conductivity. This method can effectively and conveniently compare the thermal conductivity of insulation film in MEMS. What's more, this method could be improved and optimized in further research.

\section{Corresponding Author}

Guifu Ding, gfding@sjtu.edu.cn, +86-021-34206687

\section{References}

[1]M. Asheghi, Y.K. Leung, S.S. Wong, K.E. Goodson, Appl. Phys. Lett. 71 (1997) 1798.

[2] A.D. McConnell, S. Uma, K.E. Goodson, J. Microelectromech. Syst. 10 (2001)360.

[3]Kleiner M B ..IEEE T ransact ions on Electron Devices, 1996 , 43(9).

[4]Volkein F and Baltes H .Journal of Microelectromechanical Systems , 1992 , 1(4).

[5]A. Jain, K.E. Goodson, J. Heat Transfer 130 (2008) 102402.

[6] Chang CC, Wei KH, Chang YL, Chen WC (2003) Journal of Polymer Research 10:1-6.

[7] Gonslaves KE, Xiaohe C, Marie IB. Nano Struct Mater (1997); 9(1-8):181-4. 